\title{
Hydroxychloroquine: A Potential Ethical Dilemma for Rheumatologists during the COVID-19 Pandemic
}
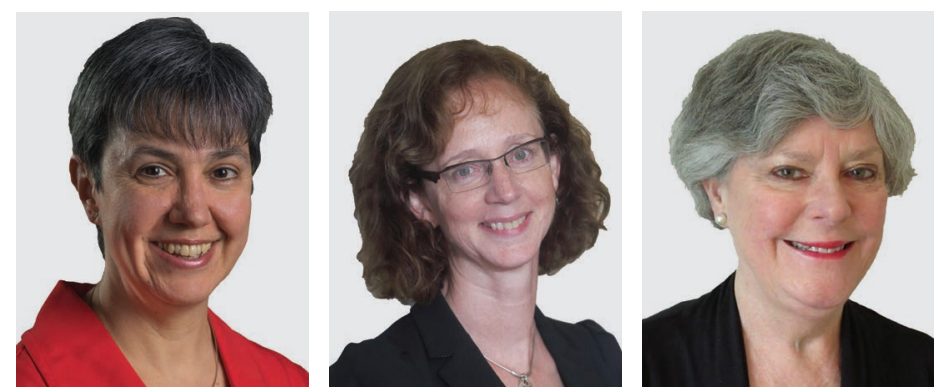

Two antimalarial agents, chloroquine (CQ) and hydroxychloroquine (HCQ), have been trusted treatments for a range of rheumatic diseases over the past 70 years $^{1}$. These agents have attracted intense media attention in the past few weeks with suggestions that this category of drugs may have potential in the management of the coronavirus (SARS-CoV2)-associated disease called COVID-19, ${ }^{2,3}$. In this unprecedented time of a world viral pandemic that is associated with considerable mortality, rheumatologists can anticipate challenges in clinical care. We must counsel patients about the appropriate use of HCQ (CQ is not currently available in Canada) in the management of rheumatic diseases, provide valid scientific information regarding the current evidence for effect in COVID-19 disease, and be poised to provide alternative drug treatment in the event that HCQ may be allocated away from routine rheumatology clinical care to treat seriously ill viral-infected patients. These issues are pertinent and imminent for the rheumatologist and require an understanding of the current available knowledge, and consideration of a possible ethical dilemma in the event that drug supply is limited.

\section{History of Antimalarial Therapy}

Antimalarial therapy began in the mid-17th century with observations by Jesuit missionaries in Peru that the bark of a specific tree, later named Cinchona, could be used to treat the severe febrile illness now know to be malaria ${ }^{4}$. In the late 17th century, the Peruvian bark, mixed with rose leaves, lemon juice, and wine was a widely used secret treatment for fever in the malarial regions of England ${ }^{4}$. Quinine is a complex structured alkaloid and the active therapeutic component of the Cinchona tree. It was isolated and named in 1820 by 2 French chemists, Pelletier and Caventou ${ }^{4}$. Until the 1850s all the Cinchona bark came from the wild forests of the Andean republics of South America, leading to world market competition. Having secretly acquired seeds and saplings from South America, botanists from Kew Gardens worked with botanical gardens in India and Java to develop hybrid species of the Cinchona tree for plantations in Java, which became the main source of quinine $e^{4}$. Following World War I, when German troops were unable to access quinine from Java, German chemists produced a synthetic version of quinine in the early 1930s named mepacrine ${ }^{4}$. Mepacrine was subsequently also produced in the United States. Enemy propaganda during World War II spread the message that mepacrine would cause yellow skin discoloration as well as infertility, resulting in mass discontinuation of malaria prophylaxis by US troops in the Far East ${ }^{4}$. In an effort to stem the tide of malaria infections in US troops, US chemists synthesized CQ, a chemical variant of sontoquine, another synthetic antimalarial produced in Germany ${ }^{4}$.

\section{Antimalarials as a Rheumatic Disease Treatment}

During World War II, there were serendipitous observations of improved autoimmune skin disease and arthritis associated with CQ use ${ }^{5}$. Toxicity related to CQ was reduced when HCQ was developed by the addition of a $\beta$-hydroxy chain to the CQ molecule ${ }^{6}$. By the 1950s, reports were emerging of the clinical use of CQ and HCQ in the management of systemic lupus erythematosus (SLE) and rheumatoid arthritis (RA) $)^{7,89}$. One such report in 1957 was by a Vancouver physician, Dr. Arthur W. Bagnall, who reported on a 4-year study of the use of CQ in 125 patients with RA who were "carefully followed up personally". With positive results in $70 \%$ of patients, Dr. Bagnall reported on the absence of "definite serious toxic effects" but did concede that there were minor toxic reactions. He also stated that there was a latent period of up to 3 months before effect, with maximum benefit for some delayed for as long as 6-12 months, leading to the statement that short duration treatment was of no value ${ }^{8}$.

Thereafter, both CQ and HCQ were identified as disease-modifying antirheumatic drugs (DMARD), with use continuing into the 21 st century. With the emergence of additional synthetic DMARD, and in recent times biological DMARD, there has likely been a reduction in use of HCQ for patients with RA in particular; but HCQ remains a mainstay of treatment for an array of rheumatic autoimmune diseases, with the most important use in SLE ${ }^{10,11}$. In a first discontinuation study, the Canadian Hydroxychloroquine Study Group, led by Dr. John Esdaile, demonstrated that stopping HCQ in patients with stable SLE resulted in over twice the

Personal non-commercial use only. The Journal of Rheumatology Copyright $(\subset) 2020$. All rights reserved. 
risk of having a disease flare ${ }^{12}$. This small pivotal study of 47 patients with stable SLE has promoted the current treatment practice, supported by guidelines worldwide, to maintain patients with SLE on HCQ indefinitely, even if disease severity requires introduction of more vigorous immunosuppressive treatments ${ }^{13,14,15}$. HCQ has proved invaluable in the management of skin manifestations of SLE as well as other immunological-related skin disorders ${ }^{16}$. There are more wide-reaching effects of HCQ with a recent study reporting attenuation of polyautoimmunity in patients with $\mathrm{SLE}^{17}$. In a nationwide Spanish study of 3679 SLE patients with 13.6\% having polyautoimmunity (comorbid autoimmune thyroiditis, RA, scleroderma, inflammatory myopathy, and mixed connective tissue disease), antimalarial drugs were associated with a lower risk of polyautoimmunity (OR $0.50,95 \%$ CI 0.38-0.67) ${ }^{17}$. An important advantage of HCQ is safety in pregnancy, first reported by Buchanan and colleagues in the United Kingdom in $1992^{18}$. In a study of 100 pregnancies in patients with SLE, specifically addressing fetal loss related to circulating antiphospholipid antibodies, no fetal malformations were associated with HCQ use ${ }^{18}$. With 3 decades of study and clinical experience, HCQ has become one of the mainstays in controlling numerous immune conditions in pregnancy without fetal risk ${ }^{19,20}$.

\section{Mechanism of Action and Recent Studies of CQ and HCQ in COVID-19}

CQ and HCQ have in vitro potential to reduce activity of SARS-CoV-2 ${ }^{2}$. They are weak bases that accumulate preferentially in the acidic environment of lysosomes and phagolysosomes with the ability to block virus infectivity by raising endosomal $\mathrm{pH}$, causing interference with virus/ cell fusion and also interfering with glycosylation of cellular receptors of SARS-CoV. Therefore, CQ and HCQ function at both the viral entry and post-entry stages of infection ${ }^{2,3}$.

Given these in vitro effects, a number of clinical trials were rapidly initiated in China to test the efficacy and safety of CQ in COVID-19-associated pneumonia. Gao and colleagues have reported early preliminary findings on more than 100 patients that CQ was superior to placebo in reducing pneumonia exacerbation, duration of illness, and duration of clearance of virus ${ }^{21}$. This report must be viewed as extremely preliminary with minimal information regarding methodology and analysis of data provided. Even so, with these early results, the National Health Commission of the People's Republic of China has included CQ in recommendations regarding management of COVID-19 pneumonia ${ }^{22}$. Colson and colleagues have suggested that $\mathrm{HCQ}$, which has a similar mechanism of action as CQ, could be equally effective with a suggested dosage of $600 \mathrm{mg} /$ day to reach serum concentrations of $1 \mu \mathrm{g} / \mathrm{ml}^{2,23}$. These researchers published a small nonrandomized study of 36 of 42 hospitalized patients who met inclusion criteria. Twenty-six received active treatment with a total $600 \mathrm{mg} /$ day of HCQ for 10 days. Six of these 26 also received azithromycin (500 $\mathrm{mg}$ for 1 day, then $250 \mathrm{mg}$ /day for 4 days) to prevent bacterial superinfection. Sixteen control patients did not receive these active treatments. Six of the HCQ patients discontinued treatment early: 3 patients were transferred to the intensive care unit (ICU), one died on Day 3, one was lost to followup, and another stopped because of nausea. No control patients were lost to followup. On Day 6, of the 20 remaining treated patients, $100 \%$ of $\mathrm{HCQ} /$ azithromycin, $57.1 \%$ HCQ only, and $12.5 \%$ of the control group showed nasopharyngeal clearance of the virus ${ }^{24}$. There are considerable limitations to this study: (1) the sample size was small; (2) the control group was recruited from other centers; (3) deaths ( 1 patient) and ICU admissions ( 3 patients) occurred early in the treatment group, and there were no clinical outcomes reported for the control group; and (4) treatment was not consistent for the active group ${ }^{24}$.

\section{Ethical Questions in the Care of Rheumatology Patients} In this rapidly evolving health context, rheumatologists find themselves placed as the most common prescribers of an "old" drug that may have potential to be a lifesaver in this catastrophic era of a viral pandemic. Although in vitro studies of antimalarials and SARS-CoV2 are promising and have a mechanistic explanation, the clinical evidence at this time is lacking and must be seen as anecdotal. Rheumatologists are not the gatekeepers for access to HCQ, but some ethical issues require consideration. It is paramount that continued clinical care of patients with chronic diseases such as SLE and inflammatory arthritis who require HCQ maintenance treatment must proceed. Discontinuation of HCQ could lead to disease flare with significant morbidity and even mortality in patients with SLE. The safety of pregnant patients with rheumatic diseases requiring treatment with HCQ must be strongly protected.

Anticipating limited availability of HCQ in the coming weeks and months, the question will be, "who should get HCQ treatment?" Rheumatologists must advocate strongly for continued access to HCQ for valid clinical indications. In addition to SLE, there are many patients with various autoimmune rheumatic diseases including palindromic rheumatism, Sjögren syndrome, cutaneous SLE, and even milder forms of RA that currently have their disease sufficiently controlled with $\mathrm{HCQ}$, not requiring treatment with more complex DMARD; they should not be denied continued treatment. Other conditions such as osteoarthritis are not indications for use of HCQ and it should not be prescribed ${ }^{25}$. Rheumatologists must join the scientific community and support the recommendation that any use of HCQ to treat COVID-19 must be limited to the hospital and/or ICU settings within the context of a formal research protocol. Therefore, off-label use outside these settings should be prohibited. Because there is no current evidence that HCQ can prevent SARS-CoV2 infection, the public should be

Personal non-commercial use only. The Journal of Rheumatology Copyright @ 2020 . All rights reserved. 
informed not to self-medicate by requesting a prescription or "borrowing" from a family member for whom it is prescribed legitimately. Importantly, patients should be educated never to share their medication. With judicious use, we hope that all those with diseases that require HCQ will continue to have access to it, and we urge producers and suppliers to be proactive in ensuring sufficient supply.

Despite these strategies, and in the event of a limited supply of HCQ, rheumatologists may have to choose which of their patients should continue taking HCQ. This ethical decision will be difficult if rheumatologists are forced to avoid prescribing HCQ. Rheumatic disease patients with life-threatening illnesses such as SLE, or pregnant patients, must have priority to continue treatment. We will need to determine for which patients with inflammatory arthritis HCQ is less essential. This may particularly apply to those whose disease is well controlled and may currently be receiving an additional DMARD. Empathetic discussion with a patient in this setting could be framed in the context of a societal contribution toward an overwhelming health disaster, with opportunity to have some influence on outcome for others. Whether this step could truly influence access and supply of HCQ as a potential treatment for this current human catastrophe is unknown. In this unprecedented time, every single effort should be made toward stemming this most awful global event, but with caution to respect and adhere to the tenets of evidence-based medicine.

\section{ROSIE SCUCCIMARRI, MD, \\ Associate Professor of Pediatrics, \\ Division of Pediatric Rheumatology, \\ McGill University Health Centre, \\ Montreal, Quebec, \\ and Chair, \\ Canadian Rheumatology Association (CRA) \\ Therapeutics Committee;}

EVELYN SUTTON, MD,

Professor of Medicine,

Division of Rheumatology,

Dalhousie University,

Halifax, Nova Scotia,

and President, CRA;

MARY-ANN FITZCHARLES, $\mathrm{MB}, \mathrm{ChB}$,

Associate Professor of Medicine,

Alan Edwards Pain Management Unit,

Division of Rheumatology,

McGill University Health Centre,

Montreal, Quebec, Canada,

and Past Chair,

CRA Therapeutics Committee.

Address correspondence to Dr. M.A. Fitzcharles, Montreal General

Hospital, McGill University Health Centre, 1650 Cedar Ave., Montreal,

Quebec H3G 1A4, Canada. E-mail: mfitzcharles@sympatico.ca

\section{REFERENCES}

1. Schrezenmeier E, Dorner T. Mechanisms of action of hydroxychloroquine and chloroquine: implications for rheumatology. Nat Rev Rheumatol 2020;16:155-66.
2. Colson P, Rolain JM, Lagier JC, Brouqui P, Raoult D. Chloroquine and hydroxychloroquine as available weapons to fight COVID-19. Int J Antimicrob Agents 2020 Mar 4 (E-pub ahead of print).

3. Wang M, Cao R, Zhang L, Yang X, Liu J, Xu M, et al. Remdesivir and chloroquine effectively inhibit the recently emerged novel coronavirus (2019-nCoV) in vitro. Cell Res 2020;30:269-71.

4. Butler AR, Khan S, Ferguson E. A brief history of malaria chemotherapy. J R Coll Physicians Edinb 2010;40:172-7.

5. Shukla AM, Wagle Shukla A. Expanding horizons for clinical applications of chloroquine, hydroxychloroquine, and related structural analogues. Drugs Context 2019;8:2019-9-1.

6. Wallace DJ. The history of antimalarials. Lupus 1996;5 Suppl 1:S2-3.

7. Mullins JF, Watts FL, Wilson CJ. Plaquenil in the treatment of lupus erythematosus. J Am Med Assoc 1956;161:879-81.

8. Bagnall AW. The value of chloroquine in rheumatoid disease: a four-year study of continuous therapy. Can Med Assoc J 1957;77:182-94.

9. Hamilton EB, Scott JT. Hydroxychloroquine sulfate ("plaguenil") in treatment of rheumatoid arthritis. Arthritis Rheum 1962;5:502-12.

10. Scherbel AL. Use of synthetic antimalarial drugs and other agents for rheumatoid arthritis: historic and therapeutic perspectives. Am J Med 1983;75:1-4

11. Lever E, Alves MR, Isenberg DA. Towards precision medicine in systemic lupus erythematosus. Pharmgenomics Pers Med 2020;13:39-49.

12. Canadian Hydroxychloroquine Study Group. A randomized study of the effect of withdrawing hydroxychloroquine sulfate in systemic lupus erythematosus. N Engl J Med 1991;324:150-4.

13. Fanouriakis A, Kostopoulou M, Alunno A, Aringer M, Bajema I, Boletis JN, et al. 2019 update of the EULAR recommendations for the management of systemic lupus erythematosus. Ann Rheum Dis 2019;78:736-45.

14. Hahn BH, McMahon MA, Wilkinson A, Wallace WD, Daikh DI, Fitzgerald JD, et al; American College of Rheumatology. American College of Rheumatology guidelines for screening, treatment, and management of lupus nephritis. Arthritis Care Res 2012; 64:797-808

15. Aouhab Z, Hong H, Felicelli C, Tarplin S, Ostrowski RA. Outcomes of systemic lupus erythematosus in patients who discontinue hydroxychloroquine. ACR Open Rheumatol 2019;1:593-9.

16. Shi H, Gudjonsson JE, Kahlenberg JM. Treatment of cutaneous lupus erythematosus: current approaches and future strategies. Curr Opin Rheumatol 2020;32:208-14.

17. Mena-Vázquez N, Fernández-Nebro A, Pego-Reigosa JM, Galindo M, Melissa-Anzola A, Uriarte-Isacelay E, et al. Hydroxychloroquine is associated with a lower risk of polyautoimmunity: data from the RELESSER Registry. Rheumatology 2019 Dec 6 (E-pub ahead of print).

18. Buchanan NM, Khamashta MA, Morton KE, Kerslake S, Baguley EA, Hughes GR. A study of 100 high risk lupus pregnancies. Am J Reprod Immunol 1992;28:192-4.

19. Gerosa M, Schioppo T, Meroni PL. Challenges and treatment options for rheumatoid arthritis during pregnancy. Expert Opin Pharmacother 2016;17:1539-47.

20. Abarientos C, Sperber K, Shapiro DL, Aronow WS, Chao CP, Ash JY. Hydroxychloroquine in systemic lupus erythematosus and rheumatoid arthritis and its safety in pregnancy. Expert Opin Drug Saf 2011;10:705-14.

21. Gao J, Tian Z, Yang X. Breakthrough: chloroquine phosphate has shown apparent efficacy in treatment of COVID-19 associated pneumonia in clinical studies. Biosci Trends 2020;14:72-3.

22. Multicenter collaboration group of Department of Science and Technology of Guangdong Province and Health Commission of Guangdong Province for chloroquine in the treatment of novel 
coronavirus pneumonia. [Expert consensus on chloroquine phosphate for the treatment of novel coronavirus pneumonia]. [Article in Chinese] Zhonghua Jie He He Hu Xi Za Zhi 2020;43:185-8

23. Lagier JC, Fenollar F, Lepidi H, Giorgi R, Million M, Raoult D. Treatment of classic Whipple's disease: from in vitro results to clinical outcome. J Antimicrob Chemother 2014;69:219-27.

24. Gautret P, Lagier JC, Parola P, Hoang VT, Meddeb L, Mailhe M, et al. Hydroxychloroquine and azithromycin as a treatment of
COVID-19: results of an open-label non-randomized clinical trial. Int J Antimicrob Agents 2020:105949.

25. Ghouri A, Conaghan PG. Treating osteoarthritis pain: recent approaches using pharmacological therapies. Clin Exp Rheumatol 2019;37 Suppl 120:124-9.

First Release May 1 2020; J Rheumatol 2020;47:783-6; doi:10.3899/jrheum.200369 